\title{
Stochastic policy-based wireless energy harvesting in green cognitive radio network
}

\author{
Hayoung Oh ${ }^{1}$ and Rong $\operatorname{Ran}^{2^{*}}$
}

\begin{abstract}
With the rapid increase of energy consumption and environment problems, the need for green techniques and harvesting energy is emerging. Network coding can provide the solution by the reduction of the unnecessary data transmission and the estimated traffic patterns. It can also amplify the synergy in an energy harvesting capable cognitive radio (CR) network since the $C R$ has the recognition and optimal decision functionalities. In this paper, we propose stochastic policy based wireless energy harvesting in green cognitive radio network. With the simulations, we show that the proposed scheme is better up to $20 \%$ of the previous work.
\end{abstract}

Keywords: Green cognitive radio network; Analog network coding; Linear network coding

\section{Introduction}

Researchers believe that one of the causes for the recent increase in carbon dioxide and serious environmental problems worldwide is the rise in the amount of data due to the advancements in information and communications technology. Yet, the effort to reduce the quantity of $\mathrm{CO}_{2}$ or data alone has not shown enough effect. Thus, a more sound communication technology should be introduced to resolve the problem. The energy harvesting capable cognitive radio (CR) network, which senses its environment and surroundings, is effective in the development of eco-friendly, low-carbon protocols since its nodes can each recognize the energy consumptions and environmental changes [1]. Previous studies on the CR network have focused on achieving high efficiency in radio frequency. However, considering the fact that the cognitive network has three stages in its process sensing the environment, deciding the optimum action, provided that a device with cognitive function can accurately sense and conserve the available frequencies and energy consumptions of different operations, it can well be applied to green, low-carbon communication protocols. Network coding reduces the amount of data and unnecessary data retransmissions and provides the estimated transmission patterns of network nodes. This technique can cut down

*Correspondence: sunnyran@ajou.ac.k

${ }^{2}$ Department of Electrical and Computer Engineering, Ajou University, Suwon, Korea

Full list of author information is available at the end of the article the consumption rate of wasted energy, thereby contributing to the enhancement of the system performance. In a linear radio network-based CR network, the patterns of data transmission and reception of a primary user (PU) can be assumed by the continuity of $k$ linear equations, and thus a secondary user (SU) can predict the duration of PU's frequency use in a huge probability $[2,3]$. As a result, in a CR network that utilizes the linear network coding technique, while the transmitted and received data of the PU is well protected, the SU can make the maximum use of vacant frequencies without wasting energy through unneeded sensing, maximizing the system performance. It is still impossible to detect PUs perfectly, so there are cases of false positive errors caused by wrong judgement of the nonexistent PU as existing and false negative errors caused by vice versa. Analog network coding is a technology that uses the interference inversely [4]. In this technique, two different nodes intentionally transmit wireless signals at the same time, and the middle node receives one integrated signal. Each source node and the nodes within the overhearing range possess the original data, so when the middle node broadcasts the integrated packet, each node destination can decode the wanted packet. This coding allows the packet to be transmitted one time less compared with the existing digital network coding [5], so it can cut the total number of packets into half compared with the uni-cast transmission method which does not use network coding technology at all. In a study by Katti et al. [4] to generate the chance for

\section{黑 Springer}

(c) 2015 Oh and Ran. This is an Open Access article distributed under the terms of the Creative Commons Attribution License (http://creativecommons.org/licenses/by/4.0), which permits unrestricted use, distribution, and reproduction in any medium, provided the original work is properly credited. 
active noise control (ANC) deliberately, the middle node had to hint (trigger) the source nodes to transmit almost simultaneously with a short delay. The reason for such process is that to integrate ANC signals, two different signals should be transmitted at once, but at the same time, the preamble of each signal should not be interfered for decoding. In addition, the previous study had not been aware of the energy harvesting and focused only on a few particular topologies to gain profits, such as Alice-Bob topology which uses the same frequency and X-topology which has a perfect overhearing range [2]. Moreover, even when two different original data were mixed on the physical layer of the middle node, one of the data should be broadcasted again to each node destination to decode the other one using changed amplitudes and phases of the wireless signal. In this paper, we suggest a technique of restoring a naturally integrated signal of $\mathrm{PU}$ and $\mathrm{SU}$ with ANC technique, using the minimum amount of energy from the CR network, which functions network coding and energy harvesting. In false negatives, PU receives some of $k$ as a linear network coded packet and the other as a mixed signal with SU packet. Even if PU's signal interferes with SU's signal, PU can still decode the packet he or she wants because the only difference the error brings is the coefficient of linear network coding packets. In false positive, SU can benefit because it can successfully transmit the packet by its own or harvest energy. However, unlike existing studies on CR network, we propose a technique for eco-friendly, low-carbonate communication by which SU can predict and recognize the frequency, the energy consumption of PU and the gain of energy harvesting, thereby choosing the optimum in false negatives or false positives. This process is modeled by partially observable Markov decision process (POMDP). In the "Related work" section, we introduce related work. In the "Stochastic policy based wireless energy harvesting in green cognitive radio network" section, we illustrate the eco-friendly, low carbonate, energy harvesting capable CR network technique which this paper proposes. Then, in the "Performance evaluation" section, we evaluate the performance. Finally, "Conclusions" section concludes the paper.

\section{Related work}

\section{Network coding}

Network coding helps the theoretical maximum network to achieve capacity by reducing the number of packet transmissions. Two specific mechanisms are involved in network coding: XOR $[5,6]$ and linear (random) network coding $[2,3]$. XOR reduces the number of transmissions since the intermediate node only broadcasts the packet once after it has been encoded from packets sent by several transmitters, rather than simply forwarding all those packets one by one. Several authors have implemented an XOR-bit-level network coding mechanism in a wireless network test-bed, showing that it improves network throughput by $38 \%$ [5]. More recently, it was shown that analog network coding [4] that utilizes signal interference rather than excludes it reduces more transmission time than the traditional bit-level network coding. All these network coding mechanisms could be widely utilized for peer-to-peer networks, efficient content distribution [7], energy efficiency [8], opportunistic routing, reliability gain, multi-hop network gain, and relay network gain, etc. In contrast, linear coding [3] operates when the intermediate nodes forward every packet that is linear transformed by a certain linear equation, and the destination nodes decode the original packets, provided they receive enough of the linear equations in an adequate number. Linear transformation is the multiplication of a vector's so-called coefficient to the bit pattern of the packet that passes through a station, and is called linear coding when the coefficient becomes 1 , whereas it is called random linear coding when the coefficient is less than 1 and larger than 0 . (Random) linear coding is generally applied with packet error recovery, a multicast scenario, and efficient delivery of urgent messages in vehicular ad hoc networks and delay-tolerant networking (DTN). Zeng et al. [9] proposed enhanced network coding (ENC) with a lower delay-energy bound on a two-way relay wireless network. In ENC, the relay transmits both coded and uncoded packets for reducing delay. Generally, in the exchange between two nodes, more energy is consumed to transmit uncoded packets. ENC is a practical algorithm used to achieve minimal average delay and a zero packet-loss rate under a given energy constraint. Dong et al. [10] provided a dynamic network coding model called dynamic network coding with packet-transmission delay guarantee (DNPDG). DNPDG effectively controls packet transmission delay in network coding by dynamically determining the coding operation and adjusting the size of the data generation. Moreover, in the coding operation, the model schedules and forwards the packets based on the measurement of the current accumulative transmission delay and the service priority of the packet. Lastly, the acknowledgement information feedback with per-hop transmission technique promotes the packet transmission of various data generations in the relay nodes. However, these schemes focused on analyzing the fundamental trade-off between average delay and data size in wireless network and did not consider the optimal energy harvesting policy as well as the network-coding-capable green cognitive radio network.

\section{Energy harvesting networks}

In a battery-powered device, the typical power management design goals are to minimize the energy consumption or to maximize the lifetime achieved while meeting 
the required performance constraints. There has been recent research effort on understanding data transmission with an energy harvesting transmitter with a rechargeable battery. In an energy harvesting node, the design target is to treat the harvested energy as a supplement to the battery energy such that the system continues to operate perennially. In [11], data transmission with energy harvesting sensors is considered, and the optimal online policy for controlling admissions into the data buffer is derived using a dynamic programming framework. In [12], energy harvesting transmitters with batteries of finite energy storage capacity are considered and the problem of throughput maximization by a deadline is solved in a static channel. In [13], offline optimal transmission policies for a single-user energy harvesting transmitter operating in a fading channel are provided. In [14], energy management policies which stabilize the data queue are proposed for single-user communication. In [15], the optimality of a variant of the back pressure algorithm using energy queues is shown. In [16], throughput optimal energy allocation is studied for energy harvesting systems in a time constrained slotted setting. In [17], information theoretically achievable rates are determined in a single-user energy harvesting communication channel. In [18, 19], minimization of the transmission completion time is considered in an energy harvesting system, and the optimal solution is obtained using a geometric framework similar to the calculus approach presented in [20]. In [21], a recursive power allocation solution for energy-efficient point-to-point machine-to-machine (M2M) communication systems with multiple energy harvesting sources is studied. They firstly formulate the problem as a nonconvex optimization problem over a finite horizon taking into account the circuit energy consumption, finite battery storage capacities, and a minimum required data rate. In [22], they focus on resource allocation algorithm design for energy-efficient communication in an orthogonal frequency division multiple access (OFDMA) downlink network with hybrid energy harvesting base station (BS). They first studied the structure of the asymptotically optimal offline resource allocation algorithm by assuming non-causal channel gain, energy arrival knowledge. It is an extension of the previous work considering an energy harvester, a constant energy source with the limited backhaul capacity [23].

\section{Wireless energy harvesting with network coding}

Up until now, most previous works assume that the relay nodes in cooperative networks are often powered by limited capacity batteries. Therefore, their lifetime is a crucial performance metric. The limited lifetime of the nodes in wireless sensor networks (WSNs) motivated researchers and system designers to study the application of energy harvesting $(\mathrm{EH})$, allowing the increase of the network lifetime by collecting energy from various sources (e.g., solar and wind). Especially, the incorporation of twoway network coding in the harvesting networks leads to significant capacity improvement by enabling the relays to process the incoming data using the XOR function before forwarding them to the respective destinations. Mekikis et al. [6] studied the performance of network coding (NC)-aided cooperative communications in large scale networks, where the relays are able to harvest energy emitted by wireless transmissions. In particular, they derive theoretical expressions for key network performance metrics, i.e., the probability of successful data exchange and the network lifetime gain [6]. Glatz et al. provide a novel framework for integrating and analyzing power-aware routing, different types of duty cycling (DC) and harvesting technology in a practical TinyOS implementation simultaneously. They implement the essential combination of energy harvesting aware routing (EHAR) together with radio and application DC and they add the novel approach of opportunistic network coding (ONC) for WSNs. They firstly give detailed analysis of the applicability of application-level DC compared to low-power MAC and power save modes for state-of-the-art WSN and harvesting system hardware [24]. In [25], the design and implementation of a networking framework for energy harvesting WSNs are presented. The main goal of the networking framework is to operate all nodes in the network in an energy neutral mode to avoid nodes exhausting the power. To achieve this goal, the networking framework implements several algorithms that can be divided into two main categories: networking and power saving. On the networking layer, the framework implements energy harvesting aware routing, network coding, and opportunistic network coding. Energy harvesting aware routing is a probabilistic routing scheme that aims to balance network traffic according to the energy profiles of the nodes. And the network coding operates alongside energy harvesting aware routing to reduce the needed packet transmissions. That is, opportunistic network coding detects the possibilities to combine packets in the data flows of the energy harvesting aware routing algorithm. For power saving, the framework implements the duty cycling of the sensor nodes and uses an existing low power listening implementation, both of which help to reduce the power that the nodes waste in idle mode. Besides, in [26], interferences resilient duty cycling in wireless sensor nodes is proposed to reduce the energy consumption. In practice, many types of wireless signals such as Zigbee, WiFi, and Bluetooh in the same frequency such as $2.4 \mathrm{GHz}$ can exist at the same time. Therefore, Zigbee signals can be broken by other signals when they wake up periodically after clear channel access (CCA) operation. Specifically, if we consider the energy consumption of the sensor node due to the false positive of the interferences (i.e., WiFi, Bluetooth, 
and electric-oven signal), the new duty cycling design with the energy harvesting function is necessary for the interferences resilience. However, these schemes focused on analyzing the fundamental energy harvesting functions with the network coding and did not consider the specific relationship between the energy harvesting and the optimal stochastic policies in the network coding capable green CR network.

\section{Stochastic policy based wireless energy harvesting in green cognitive radio network}

\section{System model}

Figure 1 shows the idle state or busy state when PU is operating based on linear network coding. SU periodically senses the energy and decides whether to transmit PU or not. However, to reduce the energy consumption, SU predicts the time remaining for complete transmission of $k$ based on the coefficient in PU's linear network coding packet. It does not sense unnecessarily during $X$ to reduce the energy consumption and harvests energy. In other words, since $k$ continuous linear network coding assumes PU's environment, SU can figure out some regularities in idle and busy states through sensing PU's frequency. The possible actions of SU after sensing PU are illustrated in Fig. 2.

Unlike previous studies, the method this paper suggests proves to be profitable since it can process ANC even in false negative states and enable SU to transmit data or harvest energy alone in false positive states. Thus, SU can transmit its single packet or harvest energy even in false positives because it does not know PU's state completely. In false negatives, the effect of ANC integration of PU and SU occurs when SU decides to transmit a single data. Therefore, it is observed that transmitting the data continuously is more beneficial than SU switching over to standby mode in any case, because at least one data is

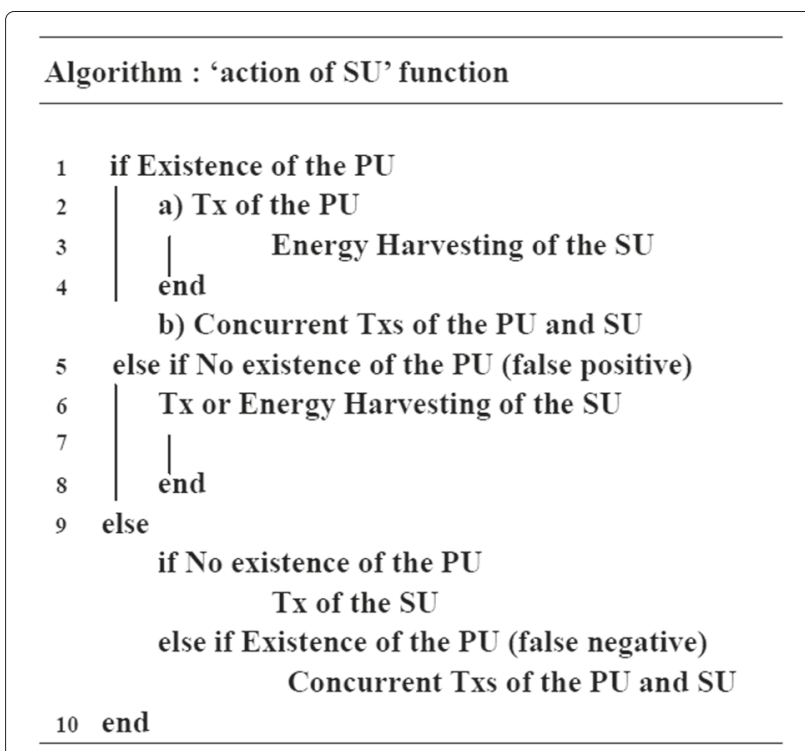

Fig. 2 The possible actions of SU after sensing PU

successfully transmitted or SU is coded with PU. And the switching energy can be saved. However, in a surrounding that considers environment and low carbon dioxide, SU simply sensing the frequency and transmitting the data unconditionally is not the best scheme. Supposing that PU uses only the energy $\left(E_{0}\right)$ previously saved in capacitor or inductor, PU's energy shows exponential decrease from the initial energy as shown in Fig. 3 [27]. This result depends on the physical characteristics of PU and the initially charged energy and has no relation with any external energy source. When time $t$ increases, the energy sharply declines. This rapid decrease in energy is called time constant and represented by the $\tau$. Thus, the time constant of $\mathrm{PU}$ is the time required for the energy to decrease at the rate of $1 / e$ and is commonly computed at $36.8 \%$ of

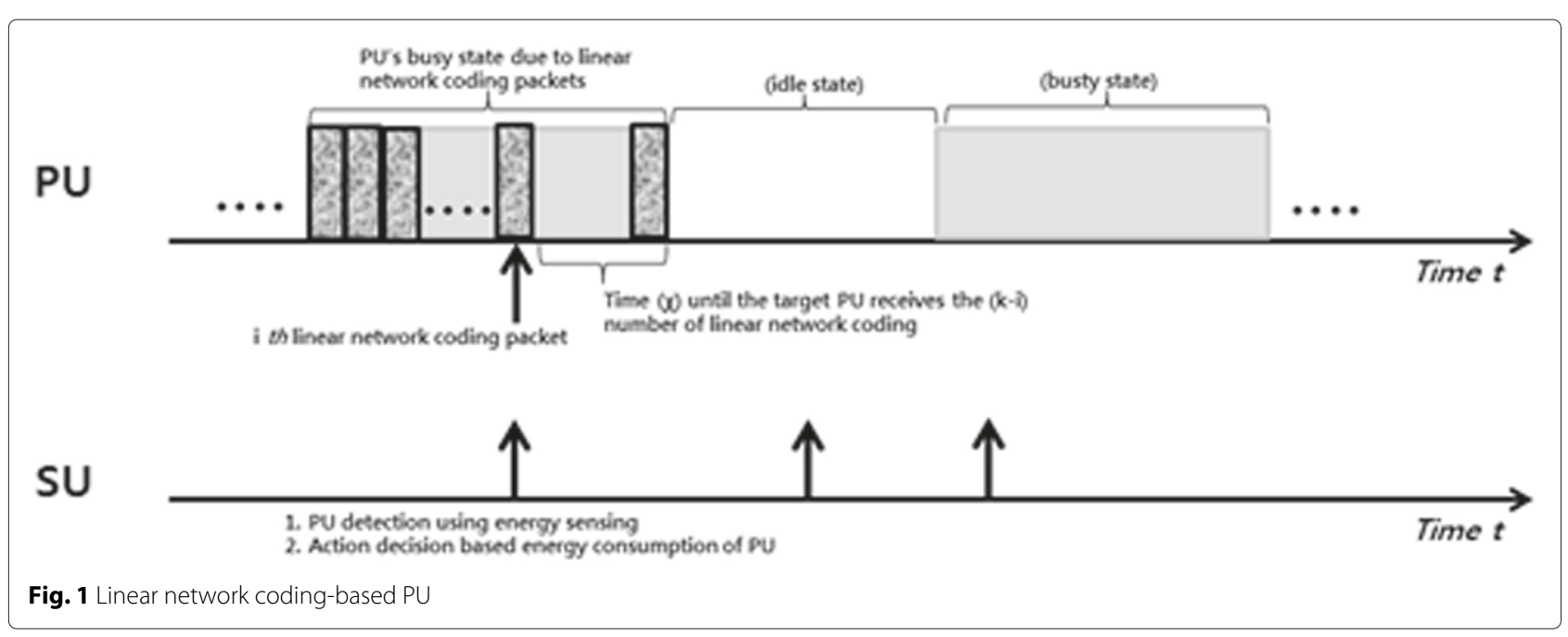




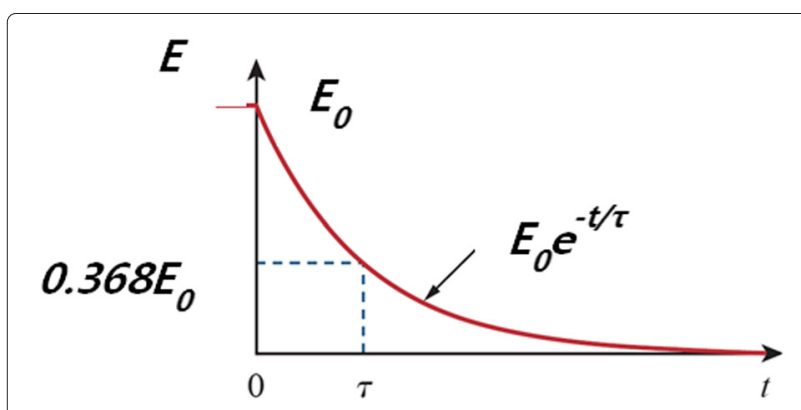

Fig. 3 Energy response function of PU

the initial value. This study sets PU's time constant as the reference value. If PU's energy consumption depending on SU's action is smaller than $36.8 \%$, ANC with the SU signal is processed for an additional decoding; if not, SU does not transmit but switch over to standby mode and harvest energy even in false positives. Thus, if the energy PU consumes when decoding the analog network coded packets is below particular value, SU chooses simultaneous transmission. If $\mathrm{SU}$ attempts to transmit under false positive state, only the packets of SU can be transmitted stably using the frequency of PU. If the energy consumed is above a particular value, SU stands by and harvests energy from the environment. If false negative states or PU is sensed properly, only the packets of PU can be transmitted stably. In other words, when $E_{\tau}$ is larger than a particular value $(\Theta)$, SU does not transmit and harvests energy even if PU's channel is sensed as idle state. The reason for this is to prevent PU's energy consumptions from being wasted when it is in false negative state.

Equation 1 shows the energy rate $\left(E_{\tau}\right)$ PU consumes depending on the action of SU. For instance, the first case is the state in which PU does exist and transmits alone, and the energy consumption rate equals the front of Eq. 1 . The $m$ th $\mathrm{PU}_{m}$ receives the other linear network coding packets for $x$ amount of time and can decode a packet it wants at last. $t_{p}$ equals 1 only when the targeted PU node destination receives the linear network coding packet $p$, $d_{p}^{l}$ represents indicator function which equals 1 only when it receives $p$ and linear network decoding is possible. $\Delta_{p}$ is the sum of the energy PU that is needed to operate for decoding during $\chi$ (actuation), the energy needed to save $k$ linear network coding packets temporarily for decoding (transient) and the energy needed for decoding (processing). The back of Eq. 1 is the second case in which the signals of SU and PU are analog network coded, and $\lambda_{p}$ is an indicator function that equals 1 only when the linear network coding packet $p$ of PU is analog network coded with the single packet of SU. $d_{p}^{a}$ represents indicator functions that equal 1 when analog network coded packet is decoded. $\Theta_{p}$ is the energy necessary for decoding the analog network coded packets.

$$
E_{\tau}\left(\mathrm{PU}_{m}\right)=\sum_{p=i}^{k}\left(\Delta_{p} t_{p} d_{p}^{l}+\Theta_{p} \lambda_{p} d_{p}^{a}\right)
$$

\section{Assumptions}

The suggested method allows SU to sense the frequency and the energy of PU and, even when error occurs, restores signals from noise as much as possible using the analog network coding technique. The following are the assumptions for the suggested method.

1. SU can check the existence or nonexistence of PU by operating the energy-sensing module.

2. SU can sense and decode the preamble of PU and figure out the coefficient and the number of the relevant packets [28].

3. Data transmission of PU occurs in continuous linear network coding (busty).

4. Even if the original packet of SU and the linear network coding packet of PU are transmitted almost simultaneously, jitter delay appears naturally in the received PU as non-synchronized part in Fig. 4. Moreover, linear network coding packets include the information of coefficients necessary for the formation of linear equation in the front or the back [3]. As a result, PU processes decoding, utilizing the coefficients that have been changed partly due to the channel and the linear equation variables it has formerly received [4]. We will further explain it in the section that discusses the practicality of the suggested method.

5. PU can combine some part of linear network coding packets that it initially received with the partially altered linear network coding packets to decode the target packet.

6. Energy consumption rate is proportional to the carbon emission rate [29].

7. SUs can harvest energy from the environment with the function module of sensors [9-12].

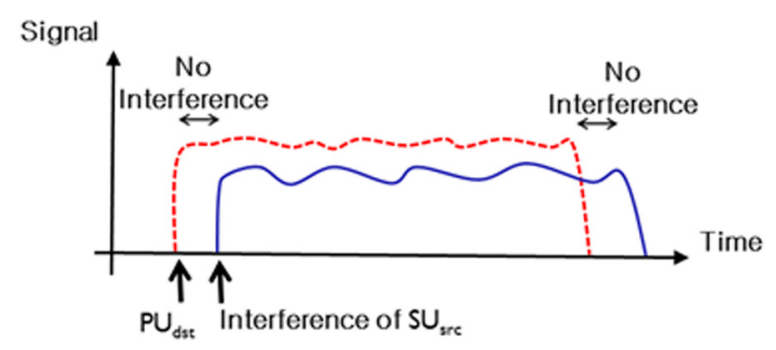

Fig. 4 The principle of the analog decoding of the PU. In case of the non-synchronized transmission, both the SU and PU packets due to the natural small jitter delay 


\section{Proposed scheme}

This paper proposes green, cognition-based energy harvesting capable communication technique for which SU decides the optimal action ("standby and harvesting"? or "transmit") considering the remaining energy of PU every moment, unlike the existent cognitive radio network techniques which considered only the recognition of frequency. To predict the transmission pattern of PU easily, we suppose PU's environment a linear network coding as described in [2]. However, when it comes to the issue of practicality, the data of PU and SU can still collide. Although it has not been dealt with in [2], this paper seeks to enhance the performance applying analog network coding technique additionally, so that advantage can be created even with the collision of the data of PU and SU. Also, it provides the foundation for eco-friendly communication technology by considering energy consumption rates for the first time in network coding based cognitive network. Figure 5 shows possible cases in the system when SU decides the optimum every moment considering the energy. Based on the observation, this paper proposes network coding based frequency and energy cognitive radio technique including energy harvesting for the first time.

\section{Discussion on the practicality of the suggested method}

Linear network coding means transmitting the packets after re-computing each into linear equations based on

If $\left(\mathrm{E}_{\mathrm{r}}<\odot\right)$

Case 1) Existence of the PU,

- Concurrent TXs of the PU and SU

Case 2) Non existence of the PU (false positive), - Tx of the SU

Case 3) Non existence of the PU.

- Tx of the SU

Case 4) Existence of the PU (false negative), - Concurrent TXs of the PU and SU

If $\left(E_{\tau}>\odot\right)$

Case 1) Existence of the PU,

- Tx of the PU and energy harvesting of SU

Case 2) Non existence of the PU (false positive), - Idle state and energy harvesting of SU

Case 3) Non existence of the PU. - Idle state and energy harvesting of SU

Case 4) Existence of the PU(false negative), - Tx of the PU and energy harvesting of SU

Fig. 5 Network coding based green cognitive radio network random coefficients. Therefore, the destination should receive enough linear equation data over $k$ to be able to decode the target packet. In other words, the destination should receive linear equations that equal the number of variables consisting of the linear equations. Random coefficients are the random figures attached to each variable when packets are made into linear equations, and they are transmitted with the data as a determinant and forms in Cramer's rule, attached in front of the packets $[3,7]$.

This paper uses minimum shifting keying modulation (MSK) as [4] does. In this modulation, when wireless signals given in complex numbers are represented by $A e^{j \Theta}$ in Phasor domain, the information is expressed with no modification in amplitude $A$ but with the change in phase $\Theta$. Furthermore, when interference occurs, the energy level of the moment when the node receives the packet increases in square of the amplitude as $A^{2}$. In result, as shown in Fig. 4, the receiver of PU measures the energy, decoding the part with regular energy change using MSK modification and separating the SU signal from PU from the part where the energy suddenly increases based on the combined vector and linear network coding packets previously received, as shown in Fig. 6. This is possible since this paper, similar to [4] which assumed that the packets involved in analog coding started with the known pilot sequence of 64 bits to distinguish the beginning of transmission, supposes that each of PU's $k$ linear network coding starts with the known sequence.

$P U_{d s t}$ receives the vectors of $P U$ and $S U$ and $X 1$ and $X 2$, with a random interval caused by the gap between small jitter delays. If $\mathrm{PU}_{\mathrm{dst}}$ receives $\mathrm{X} 1$ vector first, the amplitude of X1 vector would be $\alpha$; If $\mathrm{PU}_{\mathrm{dst}}$ receives $\mathrm{X} 2$ vector

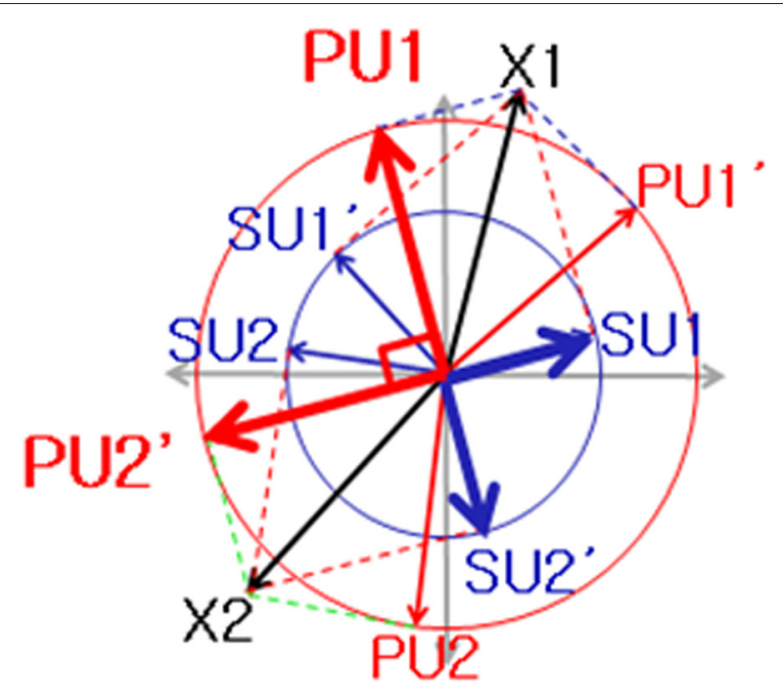

Fig. 6 The separation scheme of each SU and PU signal based on MSK when $\mathrm{PU}_{\mathrm{dst}}$ receives the combined vector 
first, the amplitude of X2 vector would be $\beta$. Accordingly, it is possible to compute in which cases are combined vectors X1 and X2 of SU and PU created, as Fig. 6. Initially received linear network coding packets enable the calculation of variable information through $\mathrm{PU}_{\mathrm{dst}}$, so it is possible to figure out that among a number of cases of phase difference in Fig. 6 with the $90^{\circ}$ gap is the signal of PU and SU.

Channel modifies only the amplitude of the signal, as shown in Fig. 6, but not the phase between the two samples. In result, there are four cases of possible signals when the receiver of PU consequently receives the vectors of PU and $\mathrm{SU}$ and X1 and X2. In MSK modification, the change in phase between the two samples is laid out by the reference of $90^{\circ}$, so we can judge that the case is each signal of SU and PU.

\section{Analysis through partially observable Markov decision}

This paper simulates modeling through partially observable Markov decision process (POMDP) to figure out which action would bring the optimal result when we know only a few parts of SU and PU in an environment where analog network coding of wireless signals of $\mathrm{PU}$ and $\mathrm{SU}$ is possible. To simulate modeling using POMDP, information on system decision epoch, state, transition probability, action, and reward is necessary. After digitally observing the system, we modeled possible states after applying the suggested method for every decision moment, depending on whether PU or SU has a residual energy or not.

The transition possibility among the states is represented by the possibility of packets of PU and SU decoded as well as the detection probability of PU. The possibility of PU's packet being decoded can be calculated by Eq. 2 .

$$
\begin{aligned}
P_{d_{p}^{p u}} & =\int_{\chi}^{\infty} \mathrm{f}(\mathrm{t}) \mathrm{dt}=1-\int_{-\infty}^{\chi} \mathrm{f}(\mathrm{t}) \mathrm{dt}=1-\mathrm{p}(X \leq \chi) \\
& =1-\left(1-e^{-\frac{1}{4} \chi}\right)=e^{-\frac{1}{4} \chi}
\end{aligned}
$$

Equation 2 shows the possibility of enough transmission of linear network coding packets over $k$ to $\mathrm{PU}_{\mathrm{dst}}$, which allows the packet of PU to be decoded. $X$ represents the remained time until $k$ linear network coding packets are collected.

The possibility of decoding the SU packet (i.e., $P_{d_{p}^{s u}}$ ) indicates every case in which SU does not judge PU as false negative. The actions SU can take are no transmission and energy harvesting or transmission. SU decides suitable action to obtain the best reward, maximization of throughput, and energy harvesting under the condition of the reciprocal of the Eq. 1, namely, the suggested scheme defines reward of the $\mathrm{SU}$ as throughput and energy harvesting gain with the reciprocal of the energy consumption rate with Eq. 1. Thereby, it aims a desirable green, low-carbonate communication in cognitive network where network coding and energy harvesting are possible.

The long-run average reward for some policy $\Theta$ is given by

$$
\mathrm{V}(\Theta)=\lim _{\mathrm{T} \rightarrow \infty} \frac{1}{T+1} Q_{\Theta}\left[\sum_{t=0}^{T} \mathrm{R}\left(Q_{t}, A_{t}\right) \mid Q_{0}=(0,0)\right]
$$

where $Q_{\Theta}$ is the expectation operator taken for the system under policy $\Theta$.

Theorem 1. There exists the optimal energy threshold $\chi_{\mathrm{PU}}^{*}$ so that the optimal deterministic action in states $(i, j)$ is to wait and harvest energy if $i \leq \chi_{\mathrm{PU}}^{*}$ and to transmit the packet if $i>$ $\chi_{\mathrm{PU}}^{*}$. To get the optimal thresholds $\chi_{\mathrm{PU}}^{*}$, we consider the long-run average reward per slot as shown in Eqs. 4 and 5.

The optimal energy threshold $\chi_{\mathrm{PU}}^{*}$ for the long-run average reward per slot is

$$
\left(\chi_{\mathrm{PU}}^{*}, *\right)=\arg \max _{\chi_{\mathrm{PU}}} R_{t x} \cdot \sigma\left(\chi_{\mathrm{PU}}, \chi_{\mathrm{SU}}\right)+R_{w}^{h} \cdot \rho\left(\chi_{\mathrm{PU}}, \chi_{S U}\right)
$$

The expected number of transmissions per slot is the states with the network coding or the PU or the SU. It can be derived by

$$
\sigma\left(\chi_{\mathrm{PU}}, \chi_{\mathrm{SU}}\right)=p_{1} \cdot \sum_{i=1}^{\chi \mathrm{PU}} \sum_{j=1}^{\chi \mathrm{SU}} \Pi_{i, j}
$$

The expected number of waiting and harvesting per slot is the states with the single packet among the neighbor nodes at the beginning of each slot. It can be derived by

$$
\begin{aligned}
\rho\left(\chi_{\mathrm{PU}}, \chi_{\mathrm{SU}}\right)= & p_{2} \cdot\left(1-p_{1}\right) \sum_{i=1, j=i+1}^{\chi \mathrm{SU}} \Pi_{i, j} \\
& +p_{2} \cdot\left(1-p_{1}\right) \sum_{i=1, j=-i}^{\chi \mathrm{SU}} \Pi_{i, j}
\end{aligned}
$$

\section{Performance evaluation}

The performance of the proposed scheme is evaluated via both analyses with Matlab s/w and ns-3 simulations. We examine the throughput performances with the energy constraints of the proposed optimal online policy and other schemes (i.e., adaptive transmission policy scheme [25], time-energy adaptive scheme [6], constant water level scheme [30], and energy adaptive scheme [31]). We perform all simulations for 1500 randomly generated realizations of the channel fade pattern. The changes in the fading level occur relatively slowly with respect to the symbol duration. In particular, we considered Rayleigh and Nakagami fading with different shape parameter. We evaluate the specified fading by sampling its probability density function with a sufficiently large number of points. Based on the extensive evaluations under various different settings, we observe that the proposed optimal policy performs very well than other schemes. Table 1 shows the parameters used in simulation. $\Psi$ is mean value of the density function of energy arrival and $\Upsilon$ is mean value of the density function of channel fading. 
Table 1 Evaluation parameters

\begin{tabular}{lc}
\hline Parameters & Values \\
\hline Frequency of 802.11 & $2.4 \mathrm{GHz}$ \\
Tx Power & $50 \mathrm{~mW}$ (i.e., $17 \mathrm{dBm}$ ) \\
Tx Rate & $500 \mathrm{~kb} / \mathrm{s}$ \\
SNR & $20-30 \mathrm{~dB}$ \\
Fading model & Rayleigh and Nakagami fading \\
Nakagami fading parameter & 3 \\
$\Psi$ & Average recharge rate with a non-negative uniform random variable \\
$\Upsilon$ & Average fading level \\
$E_{\text {max }}$ & Constraint (i.e., on the order of $1 \mathrm{~J})$ \\
The number of relay node & 100 \\
$P_{A}$ and $P_{B}$ & {$[0.1,0.9]$} \\
\hline
\end{tabular}

\section{Comparable schemes}

1. Adaptive transmission policy for short-term throughput maximization [25]: They focus on considering the problem of maximizing the transferred data in an energy harvesting node under a deadline constraint, i.e., the short-term throughput. To reflect on the adaptive transmission policy to the [25], we refer the concept of the adaptive scheme [31]. It is online energy management schemes for energy harvesting systems operating in fading channels, with finite capacity rechargeable batteries. They determined the throughput policy for deadline constrained setting under online knowledge of the events using stochastic dynamic programming in continuous time. However, the deadline constrained is a heuristic value. As a result, the optimal policy based on the deadline value is limited to maximize the throughput. Different from this work, in practice our proposed scheme considers the unsaturated scenario with the fading model in the network-coding-capable harvesting networks. Therefore, the optimal and dynamic deadline with the network coding is considered with the optimal policy in the harvest networks, i.e. the long-term throughput.

2. Time-energy adaptive scheme [6]: Our proposed policy uses the fading distribution and reacts only to the new energy arrivals and fading level changes. Therefore, it requires less feedback and less computation since they react only to the changes in the fading level. On the other hand, the timeenergy adaptive scheme harvests energy with the fixed slot periodically regards less the environment status. They rather focus on the the probability of successful data exchange for the benefits of energy harvesting in bidirectional network coded cooperative communications.

3. Constant water level scheme [30]: Our proposed scheme makes online decisions for transmit, waiting and harvesting power whenever a change in the fading level under the unsaturated scenario is observed through the average CSI in the idle slot. However, in case of the constant water level policy, it did not consider the average CSI of the previous epoch and assume that the relay node can charge the energy from the environment.

4. Energy adaptive scheme [31]: Our proposed scheme basically assumes the initial energy with the practical capacitor of the sensor nodes. And it tries to get the optimal policy in the relay node only when the residual energy is larger than the time constant threshold, $\tau$. Therefore, the exhausted energy cases rarely happen in the relay node in the proposed scheme.

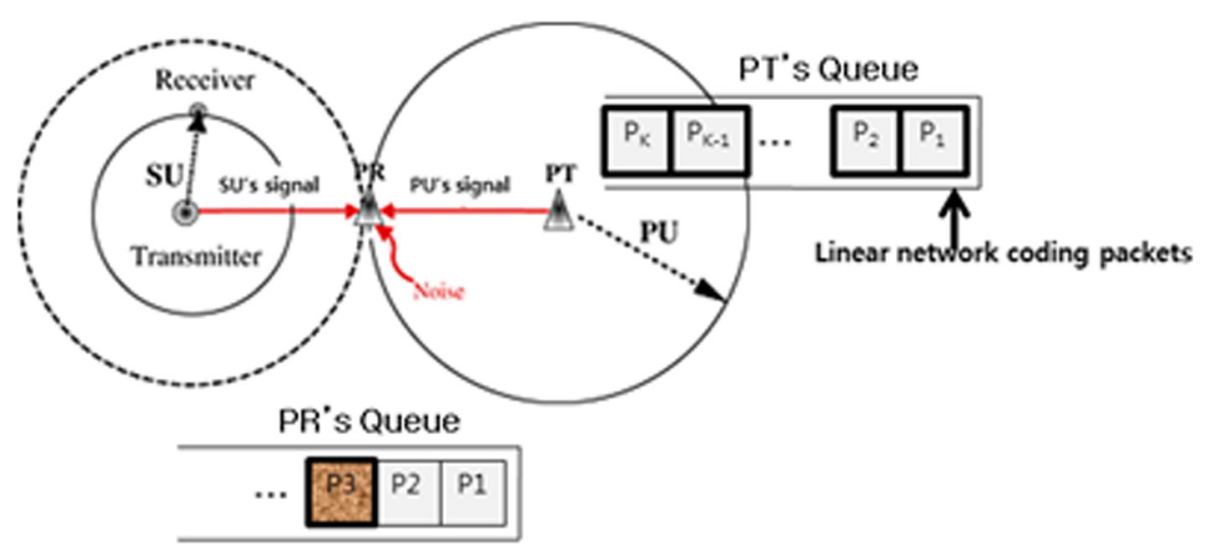

Fig. 7 Network topology 


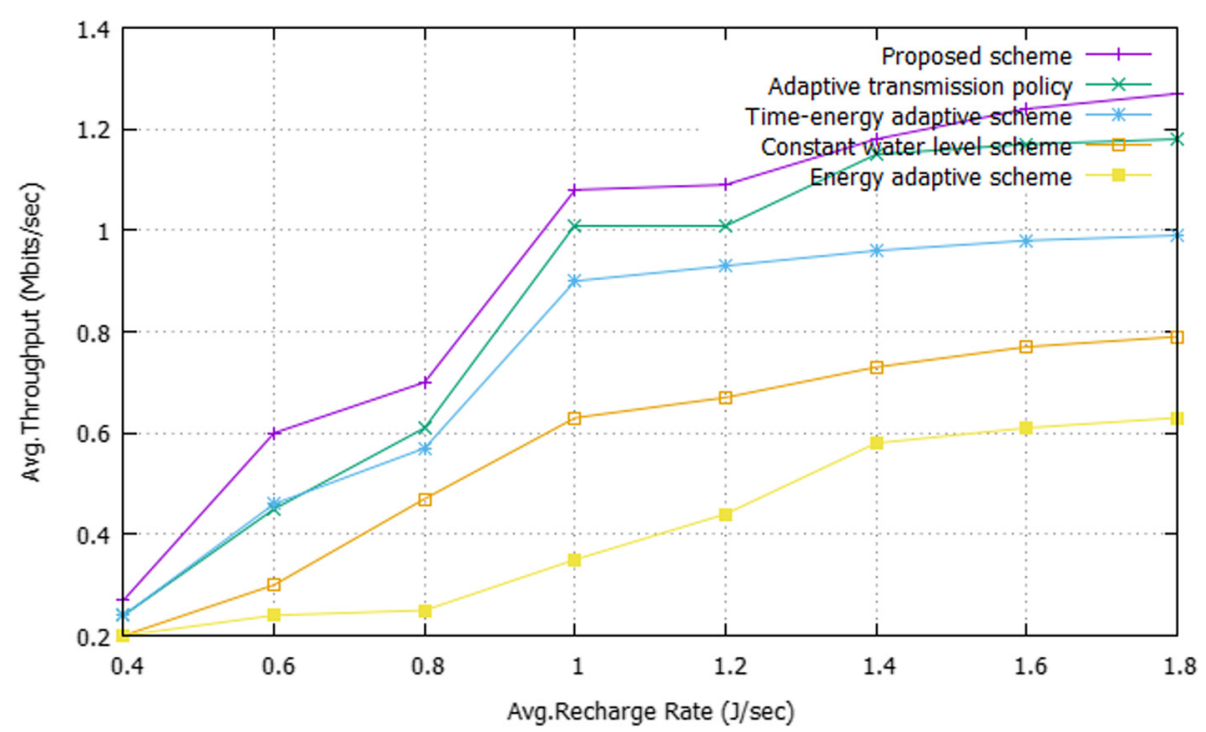

Fig. 8 Performances of the policies for different energy arrival rates under Nakagami fading with parameter $=3$ and $E_{\max }=10 \mathrm{~J}$

However, the energy adaptive scheme did not consider the importance of the relay node in the harvesting network. Therefore, bottleneck sensor nodes with no residual energy prevent the other sensor nodes from communicating.

In order to assess the performance, we consider the simple topology as shown in Fig. 7. And we define an upper bound on the performances of the policies by assuming that the channel fading levels and energy arrivals in the $[0, \mathrm{~T}]$ interval are known non-causally. In addition, the total energy in $[0, \mathrm{~T}]$ is available at the transmitter at time $t=0$. In summary, we observe that adaptive transmission policy performs quite close to the optimal online policy in the low recharge rate regime. However, its performance is viable due to the short-term throughput as the recharge rate is increased. And naturally other schemes are not better than adaptive transmission policy because of the static policy with the fading channel and energy arrival rate. In detail, we examine the setting with $T=10 \mathrm{~s}, E_{\max }=$ $10 \mathrm{~J}$ under Nakagami fading of average SNR $=5 \mathrm{~dB}$ and plot the performances in Fig. 8. As a common behavior in these settings, adaptive transmission policy performs poorer than the proposed scheme. Since the adaptive transmission policy determines the power level considering only the instantaneous energy changes, it cannot take advantage of the battery for saving energy for future use, and hence performs relatively poorly. We also simulate the performances of the policies for different

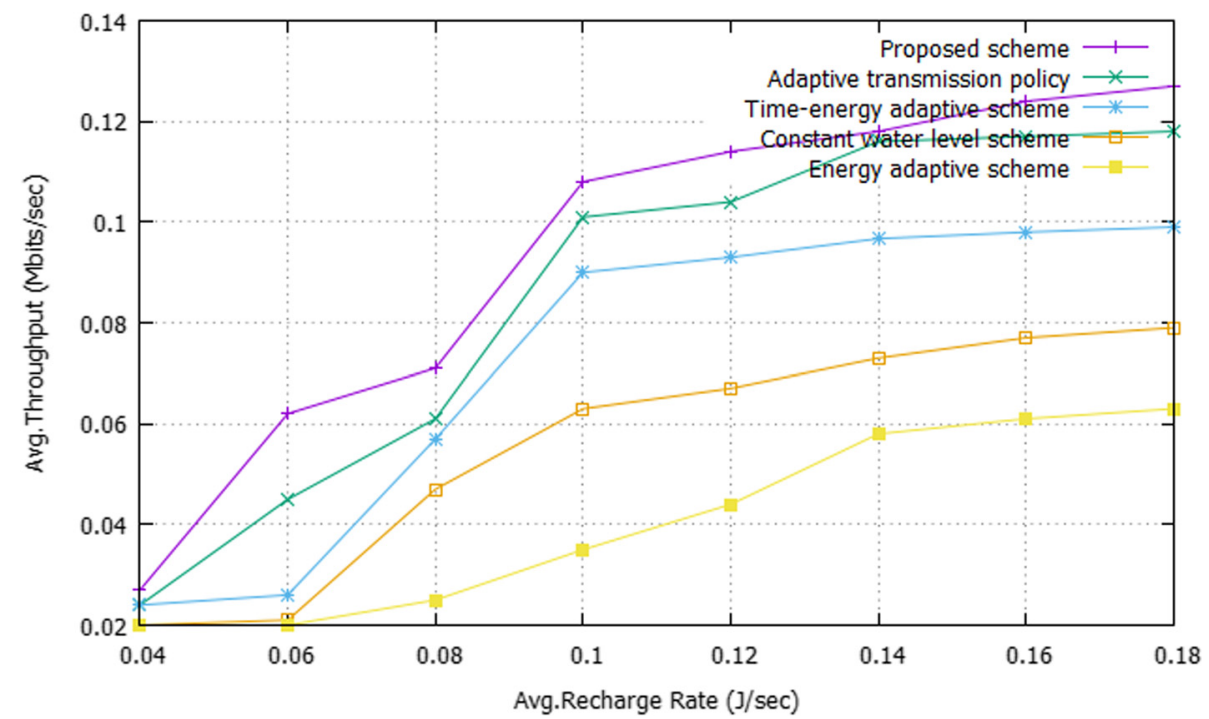

Fig. 9 Performances of the policies for different energy arrival rates under unit mean Rayleigh fading, $E_{\max }=1 \mathrm{~J}$ 
energy arrival rates under unit mean Rayleigh fading, $E_{\max }=$ $1 \mathrm{~J}, P_{A}=0.1$, and $P_{B}=0.9$ as shown in Fig. 9. In this case, the adaptive transmission policy behaves as follows: whenever a new energy arrival occurs, the policy decides to transmit with power equal to battery energy divided by the time remaining to the deadline. On the other hand, the proposed scheme decides to transmit with power equal to the average power plus battery energy divided by the time remaining to the deadline. We observe that the proposed scheme is the best.

\section{Conclusions}

Various causes of the increase in $\mathrm{CO}_{2}$ emissions emphasize the necessity of green, low-carbonate communication technique. This paper proposed a method by which we can reinforce the performance by sensing frequencies and energy in a wireless environment where network coding and energy harvesting are possible. The performance evaluation showed that the suggested technique has the highest efficiency when consuming the same amount of energy.

\section{Competing interests}

The authors declare that they have no competing interests.

\section{Acknowledgements}

This research was supported by the Basic Science Research Program through the National Research Foundation of Korea (NRF) funded by the Ministry of Science, ICT and Future Planning (Grants NRF-2012R1A1A1014392 and NRF-2014R1A1A1003562).

\section{Author details}

${ }^{1}$ Department of Electrical and Information Engineering, Soongsil University, Seoul, Korea. ${ }^{2}$ Department of Electrical and Computer Engineering, Ajou University, Suwon, Korea.

Received: 1 November 2014 Accepted: 13 May 2015

Published online: 20 June 2015

\section{References}

1. KG Shin, H Kim, AW Min, A Kumar, Cognitive radios for dynamic spectrum access: from concept to reality. IEEE Wireless Commun. 17(6), 64-74 (2010)

2. A Fanous, YE Sagduyu, A Ephremides, Reliable spectrum sensing and opportunistic access in network-coded communications. IEEE Journal on Selected Areas in Communications, Cognitive Radio Series, April. 2014

3. THo, M Medard, R Koetter, DR Karger, M Effros, S Jun, B Leong, A random linear network coding approach to multicast. IEEE Trans. Inf. Theory. 52, 4413-4430 (2006)

4. S Katti, S Gollakota, D Katabi, Embracing wireless interference: analog network coding. ACM SIGCOMM. 37(4), 397-408 (2007)

5. S Katti, H Rahul, D Katabi, WHM Médard, J Crowcroft, in Proc. of ACM SIGCOMM. XORs in the air: practical wireless network coding (Pisa, Italy, 2006)

6. PV Mekikis, AS Lalos, A Antonopoulos, L Alonso, Wireless energy harvesting in two-way network coded cooperative communications: a stochastic approach for large scale networks. IEEE. Comm. Let. 18 1011-1014 (2014)

7. I-H Hou, Y-E Tsai, T ArekAbdelzaher, I Gupta, AdapCode: adaptive network coding for code updates in wireless sensor networks. Paper presented at the 27th Conference on Computer Communications, IEEE, Phoenix, AZ, 13-18 April 2008

8. T Cui, L Chen, T Ho, Energy efficient opportunistic network coding for wireless networks, IEEE Infocom, IEEE, April 2007

9. Z Hongyi, W Chen, Delay-Energy Lower Bound on Two-Way Relay Wireless Network Coding. Technical Report. (Cornell University, 2014)

10. D Zanqiang, S Shen, LI Yangqun, Dynamic network coding with delay-guarantee in multi-hop wireless networks. J. Comput. Inform. Syst. 9(4), 1529-1538 (2013)
11. J Lei, R Yates, L Greenstein, A generic model for optimizing single-hop transmission policy of replenishable sensors. IEEE Trans. Wireless Commun. 8(2), 547-551 (2009). doi:10.1109/TWC.2009.070905

12. K Tutuncuoglu, A Yener, Optimum transmission policies for battery limited energy harvesting nodes. IEEE TWC. 11(3), 1180-1189 (2010)

13. O Ozel, K Tutuncuoglu, J Yang, S Ulukus, A Yener, Resource management for fading wireless channels with energy harvesting nodes. IEEE INFOCOM (2011) doi:10.1109/INFCOM.2011.5935203

14. V Sharma, U Mukherji, V Joseph, S Gupta, Optimal energy management policies for energy harvesting sensor nodes. IEEE Trans. Wireless Commun. 9, 1326-1336 (2010)

15. M Gatzianas, L Georgiadis, L Tassiulas, Control of wireless networks with rechargeable batteries. IEEE Trans. Wireless Commun. 9, 581-593 (2010)

16. C Ho, R Zhang, Optimal energy allocation for wireless communications powered by energy harvesters. IEEE ISIT (2010). doi:10.1109/ISIT.2010. 5513719

17. O Ozel, S Ulukus, Information theoretic analysis of an energy harvesting communication system. Workshop on Green Wireless (WGREEN) at IEEE PIMRC (2010). doi:10.1109/PIMRCW.2010.5670389

18. J Yang, S Ulukus, in 44th Annual Conference on Information Sciences and Systems. Transmission completion time minimization in an energy harvesting system (Princeton, 2010)

19. J Yang, S Ulukus, Optimal packet scheduling in an energy harvesting communication system. IEEE Trans. Comm., 60, 220-230 (2012). doi:10.1109/TCOMM.2011.112811.100349

20. M Zafer, E Modiano, A calculus approach to energy-efficient data transmission with quality-of-service constraints. IEEE/ACM Trans. Netw. 17, 898-911 (2009)

21. DWK Ng, R Schober, Energy-efficient power allocation for M2M communications with energy harvesting transmitter. Proc. IEEE Global Telecommun. Conf (2012). doi:10.1109/GLOCOMW.2012.6477832

22. DWK Ng, ES Lo, R Schober, Energy-efficient resource allocation in OFDMA systems with hybrid energy harvesting base station. IEEE Trans. Wireless Commun. 12, 3412-3427 (2013)

23. DWK Ng, ES Lo, R Schober, Energy-efficient resource allocation in multi-cell OFDMA systems with limited backhaul capacity. IEEE Trans. Wireless Commun. 11, 3618-3631 (2012)

24. PM Glatz, LB Hormann, C Steger, R Weiss, Opportunistic network coding for energy conservation in wireless sensor networks. Paper presented at the 2011 Communication Networks and Services Research Conference (CNSR), IEEE, Ottawa, ON, 2-5 May 2011

25. S Ruff, An energy harvesting networking service for wireless senso networks using network coding, Institute for Technical Informatics, 2010

26. X Zheng, in Proceedings of the 12th ACM Conference on Embedded Network Sensor Systems. ZiSense: towards interference resilient duty cycling in wireless sensor networks, SenSys 2014, (2014). doi:10.1145/2668332. 2668334

27. A Sadiku, Fundamentals of Electric Circuits, (McGraw Hill, New York, 2009)

28. K Moshksar, A Ghasemi, AK Khandani, in 2011 IEEE International Symposium on Information Theory Proceedings (ISIT). An alternative to decoding interference or treating interference as Gaussian noise (IEEE St. Petersburg, 31 July-5 August 2011)

29. MN Halgamuge, M Zukerman, K Ramamohanarao, An estimation of sensor energy consumption. Progress Electromagnetics Res. B. 12, 259-295 (2009)

30. A Goldsmith, P Varaiya, Capacity of fading channels with channel side information. IEEE. Trans. Inform. Theory. 43, 1986-1992 (1997)

31. O Ozel, K Tutuncuoglu, J Yang, S Ulukus, A Yener, Adaptive transmission policies for energy harvesting wireless nodes in fading channels. Paper presented at the 2011, CISS, IEEE, Baltimore, MD, 23-25 March 2011 